\title{
Non-Markovian master equations from entanglement with stationary unobserved degrees of freedom
}

\author{
Adrián A Budini ${ }^{1}$ and Henning Schomerus ${ }^{1,2}$ \\ ${ }^{1}$ Max Planck Institute for the Physics of Complex Systems, Nö thnitzer Straße 38, \\ 01187 Dresden, Germany \\ 2 Department of Physics, Lancaster University, Lancaster LA1 4YB, UK
}

Received 24 June 2005, in final form 2 September 2005

Published 5 October 2005

Online at stacks.iop.org/JPhysA/38/9251

\begin{abstract}
We deduce a class of non-Markovian completely positive master equations which describe a system in a composite bipartite environment, consisting of a Markovian reservoir and additional stationary unobserved degrees of freedom that modulate the dissipative coupling. The entanglement-induced memory effects can persist for arbitrary long times and affect the relaxation to equilibrium, as well as induce corrections to the quantum-regression theorem. By considering the extra degrees of freedom as a discrete manifold of energy levels, strong non-exponential behaviour can arise, such as for example power law and stretched exponential decays.
\end{abstract}

PACS numbers: $03.65 . Y z, 42.50 . L c, 03.65 . T a, 05.40 .-\mathrm{a}$

\section{Introduction}

Irreversible, dissipative quantum dynamics (such as of an open system embedded in an environment of uncontrolled degrees of freedoms) differs drastically from reversible dynamics described by a unitary time-evolution operator [1-7]. An exact description of the dissipative dynamics can be given in the projector formalism [8], which results in a master equation for the reduced density matrix. In most cases, analytic progress can only be made under the Markovian hypothesis, which requires that correlation between the system and the environment decay faster than the characteristic inverse dissipation rate $1 / \gamma$. For weak coupling, Lindblad equations can be derived which provide completely positive mapping of the density matrix from initial to final conditions; the underlying quantum dynamical semi-group can also be motivated from assumptions that are independent of the weak-coupling requirement $[5,6]$. A cornerstone of this framework is the quantum-regression theorem (QRT) [9, 7], which relates multiple-time correlation functions to single-time expectation values. Feasible exact master equations beyond the Markovian approximation are known when a spin [2-4] or a harmonic oscillator [10] is embedded in a bosonic bath. Also, transient deviations from Markovian 
behaviour on times shorter than $1 / \gamma$ are well understood [11-13]. In general, however, only few general results and manageable models are known for non-Markovian dynamics beyond the transient regime [14-19].

The few recent results about strong non-Markovian effects in quantum master equations were obtained in the context of complex environments [15], continuous time quantum random walks [16], stochastic Hamiltonians [17] and continuous measurement theory [18], as well as in more mathematical settings [19]. In general, conditions for a QRT for non-Markovian dynamics have not been established in the past.

In this paper, we present a new framework for the characterization of non-Markovian quantum system dynamics. We show that long-time non-Markovian effects naturally occur in composite environments where the system-to-reservoir coupling strength depends on additional quantum degrees of freedom. The non-Markovian behaviour arises even when the reservoir itself can be described in a Markovian approximation because the mediating degrees of freedom become entangled with the system degrees of freedom. These effects can persist for arbitrary long times, far beyond the transient regime. When the mediating degrees of freedom are eliminated, the dynamics can be rewritten as the statistical superposition of conventional master equations with a random dissipation rate. For weak coupling we obtain a class of non-Markovian Lindblad equations which preserve the complete positivity of the solution map known from the Markovian case. However, we find that the QRT in general is not fulfilled, the only exception being the approach to a stationary limit which is independent of the random dissipation rate. We also present an effective approximation to the system dynamics, which facilitates the comparison with previous results for non-Markovian dynamics. As an illustrative example we analyse the non-Markovian dispersive dynamics of a two-level system. By assuming as an extra system a manifold of quantum levels, we demonstrate the possibility of getting strong non-exponential decays.

\section{Composite environments}

We start from a full microscopic description, considering a system $\mathrm{S}$ that interacts with a composite bipartite reservoir, which consists in a bath $\mathrm{B}$ endowed with extra unobserved degrees of freedom $U$, which also participate in the system-environment interaction. The total Hamiltonian reads

$$
H_{\mathrm{T}}=H_{\mathrm{S}}+\left(H_{\mathrm{U}}+H_{\mathrm{B}}\right)+\lambda H_{\mathrm{I}}
$$

with the tripartite interaction

$$
H_{\mathrm{I}}=q_{\mathrm{S}} \otimes\left(Q_{\mathrm{U}} \otimes Q_{\mathrm{B}}\right) .
$$

The identity $\lambda H_{\mathrm{I}}=\left(\lambda Q_{\mathrm{U}}\right) \otimes\left(q_{\mathrm{S}} \otimes Q_{\mathrm{B}}\right)$ implies that the operator $Q_{\mathrm{U}}$ sets the system-reservoir coupling strength. For simplicity we assume that $Q_{\mathrm{U}}$ is a constant of motion

$$
\left[H_{\mathrm{U}}, Q_{\mathrm{U}}\right]=0 \text {. }
$$

Clearly, this assumption remains valid when the dynamics of $Q_{\mathrm{U}}$ is slower than the dissipative relaxation rate $^{3}$.

\subsection{Reformulation in terms of a random interaction parameter strength}

The dynamical evolution of the total density matrix $\rho_{\mathrm{T}}(t)$ is given by

$$
\rho_{\mathrm{T}}(t)=\exp \left[\mathcal{L}_{\mathrm{T}} t\right] \rho_{\mathrm{T}}(0),
$$

3 For very slow dynamics of $Q_{\mathrm{U}}$ (more slowly than the dissipative relaxation rate) the system will adiabatically follow the stationary state of equation (19). 
where $\mathcal{L}_{\mathrm{T}}[\bullet]=(-i / \hbar)\left[H_{\mathrm{T}}, \bullet\right]$ is the total Liouville superoperator. In order to relate these dynamics to conventional dynamics in environments with the fixed coupling strength, we eliminate the unobserved degrees of freedom $Q_{\mathrm{U}}$ for usual factorizing initial conditions $\rho_{\mathrm{T}}(0)=\rho_{\mathrm{SB}}(0) \otimes \rho_{\mathrm{U}}(0) .{ }^{4}$ The reduced density matrix $\rho_{\mathrm{SB}}(t)=\operatorname{Tr}_{\mathrm{U}}\left[\rho_{\mathrm{T}}(t)\right]$ of the system $\mathrm{S}$ and the bath $\mathrm{B}$ is then given by

$$
\rho_{\mathrm{SB}}(t)=\sum_{\mathrm{R}} P_{\mathrm{R}} \exp \left[\left(\mathcal{L}_{\mathrm{H}}+\mathcal{L}_{\mathrm{B}}+\lambda_{\mathrm{R}} \mathcal{L}_{\mathrm{I}}\right) t\right] \rho_{\mathrm{SB}}(0),
$$

where $\mathcal{L}_{\mathrm{H}}$ and $\mathcal{L}_{\mathrm{B}}$ are the Liouville operators of the system and the bath, respectively, and $\mathcal{L}_{\mathrm{I}}[\bullet]=(-i / \hbar)\left[\left(q_{\mathrm{S}} \otimes Q_{\mathrm{B}}\right), \bullet\right]$. The index $\mathrm{R}$ runs over the eigenstates $|R\rangle$ of $H_{\mathrm{U}}$. We introduced the probabilities

$$
P_{\mathrm{R}}=\left\langle R\left|\rho_{\mathrm{U}}(0)\right| R\right\rangle
$$

and the weighted coupling strengths

$$
\lambda_{\mathrm{R}}=\lambda\left\langle R\left|Q_{\mathrm{U}}\right| R\right\rangle .
$$

Indeed, equation (5) can be interpreted as a statistical average $\rho_{\mathrm{SB}}(t)=\left\langle\rho_{\mathrm{SB}}^{\mathrm{R}}(t)\right\rangle$ over solutions $\rho_{\mathrm{SB}}^{\mathrm{R}}(t)$ corresponding to a Hamiltonian

$$
H_{\mathrm{T}}^{\prime}=H_{\mathrm{S}}+H_{\mathrm{B}}+\lambda_{\mathrm{R}} q_{\mathrm{S}} \otimes Q_{\mathrm{B}}
$$

with the fixed interaction parameter $\lambda_{\mathrm{R}}$. Each solution $\rho_{\mathrm{SB}}^{\mathrm{R}}(t)$ participates with probability $P_{\mathrm{R}}$. It follows that the reduced system density matrix $\rho_{\mathrm{S}}(t)=\operatorname{Tr}_{\mathrm{B}}\left[\rho_{\mathrm{SB}}(t)\right]$ can be obtained from the reduced density matrices $\rho_{\mathrm{R}}(t)=\operatorname{Tr}_{\mathrm{B}}\left[\rho_{\mathrm{SB}}^{\mathrm{R}}(t)\right]$ by a similar average

$$
\rho_{\mathrm{S}}(t)=\sum_{\mathrm{R}} P_{\mathrm{R}} \rho_{\mathrm{R}}(t) \equiv\left\langle\rho_{\mathrm{R}}(t)\right\rangle .
$$

The random coupling formulation (9) of the system dynamics allows us to incorporate the previous knowledge about dissipative systems with the fixed coupling strength [1-14] (it may also form the basis for efficient numerical simulations). For instance, if the map $\rho_{\mathrm{R}}(0) \rightarrow \rho_{\mathrm{R}}(t)$ is completely positive then this is inherited by the map $\rho_{\mathrm{S}}(0) \rightarrow \rho_{\mathrm{S}}(t)$.

In the rest of the paper, we will use the random description to make further analytical progress for the case that the evolution of $\rho_{\mathrm{R}}(t)$ can be described by a Markovian Lindblad equation [5].

\section{Non-Markovian Lindblad equations}

When the correlation times of the bath $\mathrm{B}$ are the shortest time scale, a Markovian approximation applies. For factorizing initial conditions of the total density matrix, $\rho_{\mathrm{SB}}(0)=\rho_{\mathrm{S}}(0) \otimes \rho_{\mathrm{B}}(0)$, and weak coupling of $S$ and $B\left(\lambda_{R} \ll 1\right)$, one can then describe the evolution of the reduced density matrix $\rho_{\mathrm{R}}(t)$ by a Lindblad equation [5]

$$
\frac{\mathrm{d} \rho_{\mathrm{R}}(t)}{\mathrm{d} t}=\mathcal{L}_{\mathrm{H}}\left[\rho_{\mathrm{R}}(t)\right]+\gamma_{\mathrm{R}} \mathcal{L}\left[\rho_{\mathrm{R}}(t)\right]
$$

The random dissipation rate is given by

$$
\gamma_{\mathrm{R}}=\gamma\left\langle R\left|Q_{\mathrm{U}}^{2}\right| R\right\rangle
$$

4 For non-factorizing initial conditions between $\mathrm{S}$ and $\mathrm{U}$, the evolution of the system can be obtained by using projector operator techniques. In this case, a mapping onto a model with random dissipation rate is also possible, but initial correlations between the system and the dissipation rate have to be considered. This implies an extra inhomogeneous term in the averaged evolution equation (19), similar to the one encountered in equation (26). 
where $\gamma$ is determined by the spectral density of the environment evaluated at a characteristic frequency of the system. The Lindblad superoperator reads

$$
\mathcal{L}[\bullet]=\frac{1}{2} \sum_{\alpha}\left(\left[V_{\alpha}, \bullet V_{\alpha}^{\dagger}\right]+\left[V_{\alpha} \bullet, V_{\alpha}^{\dagger}\right]\right),
$$

with the operators $\left\{V_{\alpha}\right\}$ acting on the Hilbert space of S.

From this description, it follows that the evolution of the reduced density matrix $\rho_{\mathrm{S}}(t)=\left\langle\rho_{\mathrm{R}}(t)\right\rangle$ is non-Markovian. In fact, note that due to the statistical correlation between $\gamma_{\mathrm{R}}$ and $\rho_{\mathrm{R}}(t)$, the average of equation (10) cannot be written as a evolution which is local in time

$$
\frac{\mathrm{d} \rho_{\mathrm{S}}(t)}{\mathrm{d} t} \neq\left\{\mathcal{L}_{\mathrm{H}}+\mathcal{L}^{\prime}\right\}\left[\rho_{\mathrm{S}}(t)\right]
$$

with $\mathcal{L}^{\prime}$ being some extra Lindblad superoperator. Then, for obtaining the corresponding non-Markovian master equation, we first write the average equation (9) in the Laplace domain as

$$
\rho_{\mathrm{S}}(u)=\left\langle\frac{1}{u-\left(\mathcal{L}_{\mathrm{H}}+\mathcal{L}_{\mathrm{R}}\right)}\right\rangle \rho_{\mathrm{S}}(0) \equiv\left\langle G_{\mathrm{R}}(u)\right\rangle \rho_{\mathrm{S}}(0),
$$

where $\mathcal{L}_{\mathrm{R}}=\gamma_{\mathrm{R}} \mathcal{L}$ and $u$ is the Laplace variable. In order to cast this expression into a deterministic closed evolution equation we have to interchange the average over the random dissipation rate and the operator-inverse in the definition of $G_{\mathrm{R}}$. We employ the identity

$$
\rho_{\mathrm{S}}(u)=\frac{1}{\left\langle G_{\mathrm{R}}(u)\left[u-\left(\mathcal{L}_{\mathrm{H}}+\mathcal{L}_{\mathrm{R}}\right)\right]\right\rangle}\left\langle G_{\mathrm{R}}(u)\right\rangle \rho_{\mathrm{S}}(0),
$$

and define a deterministic superoperator $\mathbb{L}$ by demanding

$$
\rho_{\mathrm{S}}(u)=\frac{1}{u-\left[\mathcal{L}_{\mathrm{H}}+\mathbb{L}(u)\right]} \rho_{\mathrm{S}}(0) .
$$

The superoperator $\mathbb{L}$ is then determined by the condition

$$
\left\langle G_{\mathrm{R}}(u) \mathcal{L}_{\mathrm{R}}\right\rangle=\left\langle G_{\mathrm{R}}(u)\right\rangle \mathbb{L}(u)
$$

in the Laplace domain, and fulfils

$$
\left\langle G_{\mathrm{R}}(t) \mathcal{L}_{\mathrm{R}}\right\rangle=\int_{0}^{t} \mathrm{~d} \tau\left\langle G_{\mathrm{R}}(t-\tau)\right\rangle \mathbb{L}(\tau)
$$

in the time domain ${ }^{5}$.

The consequence of this procedure is a deterministic, closed, non-Markovian evolution equation of the reduced density matrix,

$$
\frac{\mathrm{d} \rho_{\mathrm{S}}(t)}{\mathrm{d} t}=\mathcal{L}_{\mathrm{H}}\left[\rho_{\mathrm{S}}(t)\right]+\int_{0}^{t} \mathrm{~d} \tau \mathbb{L}(t-\tau)\left[\rho_{\mathrm{S}}(\tau)\right]
$$

This equation has arbitrary long memory compared to the mean dissipation rate $\left\langle\gamma_{\mathrm{R}}\right\rangle$. By construction from the average of equation (10), the solution map $\rho_{\mathrm{S}}(0) \rightarrow \rho_{\mathrm{S}}(t)$ of equation (19) is completely positive.

5 The typical solution for the superoperator $\mathbb{L}(u)$ is a sum of Lindblad superoperators, each with its corresponding kernel. For the analysis of equations with a similar structure see [15-18]. 


\subsection{Quantum-regression theorem}

For Markovian Lindblad equations the QRT $[9,7]$ provides direct relations between expectation values of system observable and their correlation functions. We now use equation (19) to show that the theorem cannot be taken for granted for composite environments. Let us introduce a complete set of operators $\left\{A_{\mu}\right\}$ of the system, collected into a vector $\mathbf{A}$, and consider the expectation values

$$
\overline{\mathbf{A}(t)} \equiv \operatorname{Tr}_{\mathrm{SUB}}\left[\mathbf{A}(t) \rho_{\mathrm{T}}(0)\right],
$$

as well as the correlation functions

$$
\overline{S(t) \mathbf{A}(t+\tau)} \equiv \operatorname{Tr}_{\mathrm{SUB}}\left[S(t) \mathbf{A}(t+\tau) \rho_{\mathrm{T}}(0)\right],
$$

where $S$ is an arbitrary operator for the system. The time dependence of the operators refers to a Heisenberg representation with respect to the total Hamiltonian (1).

Based on the random formulation (9), (10) of the dynamics, the expectation values and correlation functions can be written as averages over the dissipation rate,

$$
\begin{aligned}
& \left.\overline{\mathbf{A}(t)}=\left\langle\operatorname{Tr}_{\mathrm{S}}\left[\mathbf{A} \rho_{\mathrm{R}}(t)\right]\right\rangle \equiv\langle\overline{\mathbf{A}(t)})_{\mathrm{R}}\right\rangle \\
& \begin{aligned}
\overline{S(t) \mathbf{A}(t+\tau)} & =\left\langle\operatorname{Tr}_{\mathrm{S}}\left\{\mathbf{A} \mathrm{e}^{\left(\mathcal{L}_{\mathrm{H}}+\mathcal{L}_{\mathrm{R}}\right) \tau}\left[\rho_{\mathrm{R}}(t) S\right]\right\}\right\rangle \\
& \left.\equiv\langle\overline{S(t) \mathbf{A}(t+\tau)})_{\mathrm{R}}\right\rangle
\end{aligned}
\end{aligned}
$$

The expressions deliver evolution equations

$$
\begin{aligned}
& \frac{\mathrm{d}}{\mathrm{d} t} \overline{\mathbf{A}(t)}=\left\langle\hat{\mathbf{M}}_{\mathrm{R}}{\overline{\mathbf{A}(t)_{\mathrm{R}}}}\right\rangle, \\
& \frac{\mathrm{d}}{\mathrm{d} \tau} \overline{S(t) \mathbf{A}(t+\tau)}=\left\langle\hat{\mathbf{M}}_{\mathrm{R}} \overline{S(t) \mathbf{A}(t+\tau)_{\mathrm{R}}}\right\rangle,
\end{aligned}
$$

where the matrix $\hat{\mathbf{M}}_{\mathrm{R}}$ acts on the indices of $\mathbf{A}$ and is defined by the condition

$$
\operatorname{Tr}_{\mathrm{S}}\left\{\mathbf{A}\left(\mathcal{L}_{\mathrm{H}}+\mathcal{L}_{\mathrm{R}}\right)[S]\right\}=\hat{\mathbf{M}}_{\mathrm{R}} \operatorname{Tr}_{\mathrm{S}}\{\mathbf{A} S\} .
$$

When $\gamma_{\mathrm{R}}$ is fixed, the evolution equations $(23 a)$ for expectation values and $(23 b)$ for correlation functions are identical, which recovers the QRT for Markovian dynamics. In the non-Markovian case, however, both equations still involve the average over the dissipation rate.

By using the same procedure as for the density matrix, we can transform equation ( $23 a$ ) into a closed deterministic evolution equation,

$$
\frac{\mathrm{d}}{\mathrm{d} t} \overline{\mathbf{A}(t)}=-\int_{0}^{t} \mathrm{~d} \tau \hat{\mathbf{M}}\left(t-t^{\prime}\right) \overline{\mathbf{A}\left(t^{\prime}\right)}
$$

The deterministic kernel matrix $\hat{\mathbb{M}}(t)$ fulfils an equation similar to equation (17), but written in terms of $\hat{\mathbf{M}}_{\mathrm{R}}$ and its corresponding propagator.

Equation (23b) has the same structure as equation (23a), but in the remaining average over the dissipation rate we are confronted with a subtlety. While equation $(23 a)$ is defined with initial conditions fixed at $t=0$, equation (23b) gives the solution with the initial condition $\overline{S(t) \mathbf{A}(t)}_{\mathrm{R}}$ at finite time $t$. From the definitions in equations (22b) and (24) we find statistical correlations between $\hat{\mathbf{M}}_{\mathrm{R}}$ and $\overline{S(t) \mathbf{A}(t)}_{\mathrm{R}}$, which both depend on $\gamma_{\mathrm{R}}$. Dynamically these correlations can be understood by realizing that $\overline{S(t) \mathbf{A}(t)}_{\mathrm{R}}$ is of the form of a single-time expectation value and hence evolves according to equation $(23 a)$. We still can disentangle 
the average over the dissipation rate by the procedure employed for the density matrix and the expectation value, but instead of a homogeneous equation of the form (25) obtain an inhomogeneous equation

$$
\frac{\mathrm{d}}{\mathrm{d} \tau} \overline{S(t) \mathbf{A}(t+\tau)}=-\int_{0}^{\tau} \mathrm{d} t^{\prime} \hat{\mathbb{M}}\left(\tau-t^{\prime}\right) \overline{S(t) \mathbf{A}\left(t+t^{\prime}\right)}+\mathbf{I}(t, \tau),
$$

where the term $\mathbf{I}(t, \tau)$ accounts for the correlations.

The QRT is fulfilled when the inhomogeneity $\mathbf{I}(t, \tau)$ vanishes, as is the case for Markovian dynamics where the average over the dissipation rate is absent. Equation (22b) implies that the inhomogeneity dies out in the long-time limit if the asymptotic state $\rho_{\mathrm{R}}(\infty)$ does not depend on $\gamma_{R}$; the QRT is then asymptotically valid. However, if the asymptotic state $\rho_{R}(\infty)$ depends on $\gamma_{R}$ the inhomogeneous term will contribute at all times, even in the asymptotic regime, and the QRT is invalidated.

\subsection{Effective approximation}

In order to obtain a general characterization of the dynamics, we introduce the following approximation. In equation (17) we discard the dependence introduced by the Lindblad superoperator $\mathcal{L}$ in the propagator $G_{\mathrm{R}}(u)$, i.e. $\mathcal{L}_{\mathrm{R}} \rightarrow-\gamma_{\mathrm{R}} \mathbf{I}$. Thus, we can write the approximated equation as

$$
\left\langle\frac{\gamma_{\mathrm{R}}}{u-\mathcal{L}_{\mathrm{H}}+\gamma_{\mathrm{R}}}\right\rangle \mathcal{L} \approx\left\langle\frac{1}{u-\mathcal{L}_{\mathrm{H}}+\gamma_{\mathrm{R}}}\right\rangle \mathbb{L}(u)
$$

which is solved by

$$
\mathbb{L}(u) \simeq K\left(u-\mathcal{L}_{\mathrm{H}}\right) \mathcal{L},
$$

with the function

$$
K(u)=\left\langle\frac{\gamma_{\mathrm{R}}}{u+\gamma_{\mathrm{R}}}\right\rangle\left\langle\frac{1}{u+\gamma_{\mathrm{R}}}\right\rangle^{-1}
$$

From here, the density matrix evolution reads

$$
\frac{\mathrm{d} \rho_{\mathrm{S}}(t)}{\mathrm{d} t} \simeq \mathcal{L}_{\mathrm{H}}\left[\rho_{\mathrm{S}}(t)\right]+\int_{0}^{t} \mathrm{~d} \tau K(t-\tau) \mathrm{e}^{(t-\tau) \mathcal{L}_{\mathrm{H}}} \mathcal{L}\left[\rho_{\mathrm{S}}(\tau)\right] .
$$

In this approximation all information about the extra system $U$ is encoded in the kernel $K(u)$, which is defined by equation (29). While this approximation is not controlled, it is clearly useful for characterization of the possible non-Markovian effects. The solution of equation (30) will differ from the exact solution of equation (19) only through small timedependent corrections, of order 1 , of the decay rate parameters. On the other hand, we notice that the structure of the evolution equation (30) is similar to that found in [18] in the context of a continuous measurement theory.

\subsection{Stochastic state representation}

In the previous approximation, it is not clear whether the final evolution guarantees the completely positive condition. Here, by introducing a stochastic representation of the dynamics, we prove that indeed this condition is preserved.

In the Laplace domain equation (30) reads

$$
u \rho_{\mathrm{S}}(u)-\rho_{\mathrm{S}}(0)=\left\{\mathcal{L}_{\mathrm{H}}+K\left(u-\mathcal{L}_{\mathrm{H}}\right) \mathcal{L}\right\} \rho_{\mathrm{S}}(u) .
$$


By assuming that $\mathcal{L}=\mathcal{E}-\mathrm{I},{ }^{6}$ with $\mathcal{E}[\bullet]=\sum_{\alpha} V_{\alpha} \bullet V_{\alpha}^{\dagger}$, we arrive at

$$
\rho_{\mathrm{S}}(u)=\left\{\frac{1}{\mathrm{I}-w\left(u-\mathcal{L}_{\mathrm{H}}\right) \mathcal{E}}\right\} \frac{1-w\left(u-\mathcal{L}_{\mathrm{H}}\right)}{u-\mathcal{L}_{\mathrm{H}}} \rho_{\mathrm{S}}(0),
$$

where we have introduced the function

$$
w(u)=\left\langle\frac{\gamma_{\mathrm{R}}}{u+\gamma_{\mathrm{R}}}\right\rangle .
$$

From here, it is possible to obtain the formal solution

$$
\rho_{\mathrm{S}}(t)=P_{0}(t) \mathrm{e}^{t \mathcal{L}_{\mathrm{H}}} \rho_{\mathrm{S}}(0)+\int_{0}^{t} \mathrm{~d} \tau w(t-\tau) \mathrm{e}^{(t-\tau) \mathcal{L}_{\mathrm{H}}} \mathcal{E}\left[\rho_{\mathrm{S}}(\tau)\right],
$$

where $P_{0}(u)=[1-w(u)] / u=\left\langle\left(u+\gamma_{\mathrm{R}}\right)^{-1}\right\rangle$. This equation has a clear stochastic interpretation. It corresponds to an average of a stochastic density matrix $\rho_{\mathrm{st}}(t)$ whose evolution consists in the application, at random times, of the superoperator $\mathcal{E}$, while in the intermediate intervals the system state evolves with its unitary evolution $\exp \left[t \mathcal{L}_{\mathrm{H}}\right]$. The statistics of the random times is dictated by $w(\tau)$, which can be interpreted as a waiting time distribution, i.e. it is the probability density for an interval $\tau$ between consecutive applications of $\mathcal{E}$. In correspondence, $P_{0}(t)=1-\int_{0}^{t} \mathrm{~d} \tau w(\tau)$ is the survival probability associated with $w(\tau)$. Then, the first term in equation (34) represents realizations without any application of the superoperator $\mathcal{E}$, while the integral term accounts for all other realizations. Thus, we can write $\rho_{\mathrm{S}}(t)=\left\langle\left\langle\rho_{\mathrm{st}}(t)\right\rangle\right\rangle$, where $\langle\langle\cdots\rangle\rangle$ denotes the average over the random times at which $\mathcal{E}$ is applied. As each realization preserves the complete positivity, this property is also present in the averaged evolution.

The previous stochastic framework allows us to clarify the role of the kernel $K(t)$. This follows after introducing the sprinkling distribution [20] $f(t)=w(t) \theta(t)+\int_{0}^{t} w(t-\tau) f(\tau)$, where $\theta(t)$ is the step function ${ }^{7}$. From its definition, $f(t)$ gives the probability density for an event at time $t$, disregarding the possibility of extra events in $(0, t)$. In the Laplace domain it reads $f(u)=w(u) /[1-w(u)]$. From here it is simple to get the relation $K(t)=\mathrm{d} f(t) / \mathrm{d} t$, which defines the kernel as the rate of the sprinkling distribution.

When the unitary dynamics commutate with the action of the superoperator $\mathcal{E}$, in an interaction representation the stochastic dynamics reduces to that presented in [16]. On the other hand, a similar stochastic interpretation may be proposed for the exact evolution equation (19), involving many renewal processes. Nevertheless, their specific form depends on the details of each problem.

It is interesting to note that expressions similar to equation (34) arise in the context of the micromaser theory [21-23]. This system consists in an electromagnetic cavity that is continuously pumped with excited atoms. In our scheme, the waiting time distribution $w(t)$ can be associated with a non-Poissonian pump statistic, while the superoperator $\mathcal{E}$ with the transformation produced in the cavity field by the passage of each atom. Therefore, the extra system $U$ can be associated with the pumps degree of freedom. This comparison enlightens the dynamical origin of the non-Markovian effects.

6 In a general case, where $\mathcal{L} \neq \mathcal{E}-\mathrm{I}$, with $\mathcal{E}$ a completely positive superoperator, the stochastic dynamics can only be established in a formal way after introducing a limit procedure [16]. In this case, the superoperator reads $\mathcal{E}[\rho]=$ $\left\{\mathrm{I}+\left[\mathrm{e}^{\kappa \mathcal{L}}-\mathrm{I}\right]\right\} \rho$, where $\kappa$ must be intended as a control parameter. Equation (30) is recovered in the limit in which simultaneously $\kappa \rightarrow 0$ and the number of events by unit of time go to infinite, the last limit being controlled by the sprinkling distribution $f(t) \rightarrow f(t) / \kappa[20]$ associated with the waiting time distribution $w(t)$. Then, while the stochastic dynamics can be formally extended to the general case, it is not possible to implement it as a numerical method to solve the corresponding master equation.

7 Here the step function $\theta(t)$ is defined as $\theta(t)=1$ for $t>0$, and $\theta(t)=0$ for $t \leqslant 0$. With this definition it follows that $\left.f(t)\right|_{t=0}=0$. 


\subsection{Dephasing of a two-level system}

As an illustrative example of our results we consider a two-level system, described by the Hamiltonian $H_{\mathrm{S}}=(1 / 2) \hbar \omega_{\mathrm{A}} \sigma_{z}$, where $\sigma_{z}$ is the Pauli $z$-matrix, which is weakly connected to a composite environment. The reservoir B is described by the dispersive Lindblad operator

$$
\mathcal{L}[\bullet]=\frac{1}{2}\left(\left[\sigma_{z} \bullet, \sigma_{z}\right]+\left[\sigma_{z}, \bullet \sigma_{z}\right]\right),
$$

and the mediating part $\mathrm{U}$ is described by an arbitrary set $\left\{\gamma_{\mathrm{R}}, P_{\mathrm{R}}\right\}$ of dissipation rates and weights.

The evolution of the density matrix is given by the non-Markovian Lindblad equation (19). From equation (17) we find the superoperator $\mathbb{L}(u)=K\left(u-\mathcal{L}_{\mathrm{H}}\right) \mathcal{L}$, with the kernel $K(u)$ defined in equation (29). Thus, in this case, the evolution equation (30) is exact.

The density matrix solution can be easily found in an interaction representation with respect to the system Hamiltonian. We find the completely positive map

$$
\begin{aligned}
& \rho_{\mathrm{S}}(t)=g_{+}(t) \rho_{\mathrm{S}}(0)+g_{-}(t) \sigma_{z} \rho_{\mathrm{S}}(0) \sigma_{z}, \\
& g_{ \pm}(t)=\frac{1}{2}\left[1 \pm P_{0}(t)\right] .
\end{aligned}
$$

The function $P_{0}(t)=\sum_{\mathrm{R}} P_{\mathrm{R}} \exp \left[-\gamma_{\mathrm{R}} t\right]$ is the survival probability defined previously. Depending on the distribution of the dissipation rate, arbitrary forms of the decay can be obtained from this average over exponential functions. Hence, the non-Markovian behaviour can be observed in the relaxation of the density matrix to the stationary state.

Let us now illustrate the consequences for the QRT; hence, the expectation values and correlators of the vector of Pauli operators $\mathbf{A} \equiv\left\{\sigma_{x}, \sigma_{y}, \sigma_{z}, I\right\}$. For the expectation value (in the interaction representation) we find

$$
\overline{\mathbf{A}(t)}=\hat{\mathbb{G}}(t) \overline{\mathbf{A}(0)},
$$

where the matrix propagator reads $\hat{\mathbb{G}}(t)=\operatorname{diag}\left\{P_{0}(t), P_{0}(t), 1,1\right\}$. Consistently with equation (26), the correlation functions

$$
\overline{S(t) \mathbf{A}(t+\tau)}=\hat{\mathbb{G}}(\tau) \overline{S(t) \mathbf{A}(t)}+\mathbf{I}_{0} h(t, \tau) .
$$

feature an extra inhomogeneity, which is given by $\mathbf{I}_{0}=\hat{\mathbb{D}} \operatorname{Tr}_{S}\left\{\left[\rho_{\mathrm{S}}(0)-\rho_{\mathrm{S}}(\infty)\right] S \mathbf{A}\right\}$, with the matrix $\hat{\mathbb{D}}=\operatorname{diag}\{1,1,0,0\}$, and the function $h(t, \tau)=P_{0}(t+\tau)-P_{0}(t) P_{0}(\tau)$. In the Markovian case $h$ vanishes since the survival probability $P_{0}(t)$ is an exponential, and the QRT is valid for all times. For non-Markovian dynamics, the theorem is valid in the long-time asymptotic, since the inhomogeneous term dies out as the equilibrium state $\rho_{\mathrm{S}}(\infty)$ is attained (consistently, in this example $\rho_{\mathrm{R}}(\infty)$ is independent of the dissipation rate $\gamma_{\mathrm{R}}$ ).

\section{Entanglement with a discrete manifold of energy levels}

Up to now we have left unspecified the unobserved degrees of freedom $U$, which determine the set $\left\{\gamma_{\mathrm{R}}, P_{\mathrm{R}}\right\}$. In this section, we will analyse the case in which $\mathrm{U}$ is defined by a discrete set of energy levels.

In the effective approximation, the properties of the unobserved degree of freedom are introduced through the kernel $K(t)$, which is associated with the renewal process defined by $w(t)=\sum_{\mathrm{R}} P_{\mathrm{R}} \gamma_{\mathrm{R}} \mathrm{e}^{-\gamma_{\mathrm{R}} t}$. This process has two characteristic times scales

$$
\langle\gamma\rangle=\sum_{\mathrm{R}} P_{\mathrm{R}} \gamma_{\mathrm{R}}, \quad\langle\tau\rangle=\sum_{\mathrm{R}} P_{\mathrm{R}} \gamma_{\mathrm{R}}^{-1} .
$$

When these constant are well defined, the average rate $\langle\gamma\rangle$ defines an exponential decay of the waiting time distribution at short times $[\langle\gamma\rangle t<1], \lim _{u \rightarrow \infty} w(u) \approx\langle\gamma\rangle /(u+\langle\gamma\rangle)$, and the 
average waiting time $\langle\tau\rangle=\int_{0}^{\infty} \mathrm{d} t w(t)$ characterizes an exponential decay of $w(t)$ in a long time regime $[t>\langle\tau\rangle], \lim _{u \rightarrow 0} w(u) \approx 1-u\langle\tau\rangle$. On the other hand, in terms of the sprinkling distribution we get $\lim _{t \rightarrow 0^{+}} f(t)=\langle\gamma\rangle$ and $\lim _{t \rightarrow \infty} f(t)=1 /\langle\tau\rangle$.

\subsection{Entanglement with a two-state system}

First we assume that the unobserved system $U$ is a two-level system. The waiting time distribution then reads $w(t)=P_{\uparrow} \gamma_{\uparrow} \mathrm{e}^{-\gamma_{\uparrow} t}+P_{\downarrow} \gamma_{\downarrow} \mathrm{e}^{-\gamma_{\downarrow} t}$, with the condition $P_{\uparrow}+P_{\downarrow}=1$ and arbitrary rates $\gamma_{\uparrow / \downarrow}$. Introducing the rates $\eta=P_{\uparrow} \gamma_{\downarrow}+P_{\downarrow} \gamma_{\uparrow}$ and $\beta=\left[\left\langle\gamma^{2}\right\rangle-\langle\gamma\rangle^{2}\right] /\langle\gamma\rangle$, the Laplace transform $w(u)$ can be written as

$$
w(u)=\frac{\langle\gamma\rangle}{u+\langle\gamma\rangle+\beta \sigma(u)},
$$

with $\sigma(u)=u /[u+\eta /(\langle\gamma\rangle\langle\tau\rangle)]$. The corresponding kernel equation (29) results as

$$
K(u)=\frac{\langle\gamma\rangle}{1+\beta \sigma(u) / u},
$$

which in the time domain reads $K(t)=\langle\gamma\rangle\left[\delta(t)-\beta \mathrm{e}^{-\eta t}\right]$. We note that the fluctuation rate $\beta$ controls departure from a Markov kernel. The sprinkling distribution results as $f(t)=\langle\gamma\rangle \theta(t)-\left[\langle\gamma\rangle-\langle\tau\rangle^{-1}\right]\left(1-\mathrm{e}^{-\eta t}\right)$, where we have used $\eta\left[1-(\langle\gamma\rangle\langle\tau\rangle)^{-1}\right]=\beta$. As expected, $\langle\gamma\rangle$ and $\langle\tau\rangle^{-1}$ give respectively the asymptotic values of $f(t)$ in the short and long time regimes, while $\eta$ gives the rate for the transition between these two regimes.

\subsection{Entanglement with an $N$-manifold of states}

Now we characterize the case in which the system $U$ consists in a manifold of $N$ states $[0 \leqslant R \leqslant N-1]$ whose consecutive energy difference is constant. We assume that the coupling strength of each level with the system-bath-set decreases in an exponential way with the level energies, as well as their stationary populations

$$
\gamma_{\mathrm{R}}=\gamma \exp [-b R], \quad P_{\mathrm{R}}=\frac{\left(1-\mathrm{e}^{-a}\right)}{\left(1-\mathrm{e}^{-a N}\right)} \exp [-a R] .
$$

The constant $\gamma$ characterizes the Markovian decay of the system, and $b$ is a free dimensionless parameter that measures the strength of the coupling between each states and the systembath-set, i.e. $Q_{\mathrm{U}}=\sum_{R=0}^{N-1} \exp [-b R / 2]|R\rangle\langle R|$. The second free parameter $a$ measures the exponential decay of the populations. By taking $a=\hbar \omega_{0} / k T$, where $\hbar \omega_{0}$ is the difference of energy between consecutive levels, the populations correspond to a thermal distribution at temperature $T$.

The average rate reads

$$
\langle\gamma\rangle=\gamma\left(\frac{1-\mathrm{e}^{-a}}{1-\mathrm{e}^{-(a+b)}}\right)\left(\frac{1-\mathrm{e}^{-(a+b) N}}{1-\mathrm{e}^{-a N}}\right),
$$

from where the average waiting time follows immediately

$$
\langle\tau\rangle=\gamma^{-1}\left(\frac{1-\mathrm{e}^{-a}}{1-\mathrm{e}^{-(a-b)}}\right)\left(\frac{1-\mathrm{e}^{-(a-b) N}}{1-\mathrm{e}^{-a N}}\right) .
$$

In an intermediate regime, $\langle\gamma\rangle^{-1}<t<\langle\tau\rangle^{-1}$, the corresponding waiting time distribution may present a power law behaviour. In fact, in the limit of $N \rightarrow \infty$, where the $N$-manifold states are equivalent to a thermal harmonic oscillator in equilibrium at temperature $T$, it is 
possible to prove that, after a time transient of order $1 / \gamma$, the waiting time distribution behaves as [24]

$$
w(t) \approx 1 /(\gamma t)^{1+\alpha}, \quad \alpha=a / b .
$$

For finite $N$, this behaviour is also present, nevertheless the asymptotic behaviour changes to an exponential decay with rate $\langle\tau\rangle^{-1}$.

For $a<b[0<\alpha<1]$ the behaviour of $w(t)$ can be captured with a simple analytical expression. Taking into account the results for the case $N=2$, equations (40), (41), we propose the complete monotone function [16]

$$
w(u)=\frac{\langle\gamma\rangle}{u+\langle\gamma\rangle+\beta^{1-\alpha} \sigma_{\alpha}(u)},
$$

where as before $\beta \approx\left[\left\langle\gamma^{2}\right\rangle-\langle\gamma\rangle^{2}\right] /\langle\gamma\rangle$, and now $\sigma_{\alpha}(u)=\left[\left(u+\gamma_{\mathrm{c}}\right)^{\alpha}-\gamma_{\mathrm{c}}^{\alpha}\right]$. Using the relation $K(u)=w(u) / P_{0}(u)$, the kernel reads

$$
K(u)=\frac{\langle\gamma\rangle}{1+\beta^{1-\alpha} \sigma_{\alpha}(u) / u} .
$$

From $\lim _{u \rightarrow 0} w(u) \approx 1-\left[u+\beta^{1-\alpha} \sigma_{\alpha}(u)\right] /\langle\gamma\rangle$, the asymptotic exponential decay of $w(t)$ with rate $\langle\tau\rangle^{-1}$ can be fitted with the cutoff $\gamma_{\mathrm{c}}$ after imposing the relation $\alpha\left(\beta / \gamma_{\mathrm{c}}\right)^{1-\alpha}=\langle\gamma\rangle\langle\tau\rangle-1$. On the other hand the constant $\beta$ fits the power law regime. In fact, in the limit $N \rightarrow \infty$, the average waiting time equation (44) is infinite, which implies $\gamma_{\mathrm{c}}=0$. Thus, $\lim _{u \rightarrow 0} w(u) \approx 1-\beta^{1-\alpha} u^{\alpha} /\langle\gamma\rangle$, implying a pure power law asymptotic behaviour [25].

The previous analysis demonstrates that the transient behaviour between the short and asymptotic exponential decays of $w(t)$ is described by a power law. As we have seen in the previous section, this behaviour is in general reflected by the system dynamics. On the other hand, we notice that when $N \rightarrow \infty$, by maintaining the average rate $\langle\gamma\rangle$ fixed, the fluctuation rate $\beta$ reaches its maximum value in the limit of both, strong coupling $b \ll 1$ and small populations decay $a \ll 1$ (which can also be read as a high temperature limit). In this case, after the transient $t\left\langle\langle\gamma\rangle^{-1}\right.$, the waiting time distribution and the kernel can be approximated by the expressions

$$
w(u) \approx \frac{A_{\alpha}}{A_{\alpha}+u^{\alpha}}, \quad K(u) \approx A_{\alpha} u^{1-\alpha},
$$

with $A_{\alpha} \approx\langle\gamma\rangle / \beta^{1-\alpha} \approx\langle\gamma\rangle^{\alpha}$. These expressions correspond to a fractional derivative evolution [16], where stretched exponential and power law behaviour arises jointly.

\section{Conclusions}

We have shown that a system in a composite bipartite environment (where the system-toreservoir coupling depends on other degrees of freedom) follows non-Markovian dynamics even when the reservoir itself can be eliminated by a Markovian approximation. The nonMarkovian effects originate in the entanglement of the system with the mediating degrees of freedom, and may persist for arbitrary long times.

Our results are derived from a random rate reformulation of the dynamics in the composite environment which allows us to make full contact to the established theory of dissipative systems with constant coupling. On this basis, we formulated non-Markovian Lindblad equations which provide complete positive mappings of the density matrix from initial to final conditions, and identified conditions for the quantum-regression theorem. It should be noted that the random rate formulation is not restricted to the Lindblad master equations but can 
be applied to other master equations, including exact master equations which may already include non-Markovian effects at fixed coupling.

In an effective approximation all information about the extra degrees of freedom is introduced by a memory kernel. The corresponding density matrix evolution can be interpreted in terms of the stochastic process in the system Hilbert space. System decay behaviours ranging from stretched exponential to power law can be obtained by taking the system $U$ as a discrete manifold of states.

The present formalism substantiates previous results on non-Markovian master equations [14-19], and puts them into the alternative and greater perspective of systems embedded in a composite environment. Our motivation to study this kind of environment arises from recent experiments on fluorescent single quantum dots [26-29], where non-Markovian effects on time scales much larger than $1 / \gamma$ were found. While the underlying physical mechanisms are not completely clear, it has been argued $[30,31]$ that the experimental results can only be recovered when one accounts for additional degrees of freedom which modulate the dissipative coupling. Thus, besides its theoretical interest in the context of strong non-Markovian effects in open quantum systems, the characterization of the dynamics induced by composite environments may also be of interest in those experiments. On the other hand, we believe that the present results may be useful in modelling the dynamics of open quantum systems embedded in complex structured host environments. In fact, the tri-partite interaction investigated by us naturally arises when one considers the dynamical effects of a disordered condensed-matter environment on a system coupled to a (say, Markovian) environment. The coupling strength of the system to the Markovian environment is proportional to the density of states of the Markovian environment at a characteristic frequency of the system; this density of states, in turn, depends on the condensed-matter environment (e.g. the disorder configuration, or the charging of trap states). Taking the dynamics of the condensed-matter environment into account, the coupling strength then becomes as a dynamical variable, leading to the multiplicative tri-partite coupling that was considered in this paper.

In the description of condensed-matter systems, multipartite interactions also frequently appear as a consequence of a suitable transformation (such as the Fröhlich transformation) [32]. Such transformations can be used to eliminate (at least to some order of a small parameter) the time dependence of a certain subsystem, which then takes on the characteristics of our (stationary) unobserved degrees of freedom.

\section{References}

[1] Zurek W H 2003 Rev. Mod. Phys. 75715

[2] Grifoni M and Hänggi P 1998 Phys. Rep. 304229

[3] Weiss U 1999 Quantum Dissipative Systems (Singapore: World Scientific)

[4] Leggett A J, Chakravarty S, Dorsey A T, Fisher M P, Garg A and Zwerger W 1987 Rev. Mod. Phys. 591

[5] Alicki R and Lendi K 1987 Quantum Dynamical Semigroups and Applications (Lecture Notes in Physics vol 286) (Berlin: Springer)

[6] Nielsen M A and Chuang I L 2000 Quantum Computation and Quantum Information (Cambridge: Cambridge University Press)

[7] Carmichael H J 1993 An Open Systems Approach to Quantum Optics (Lecture Notes in Physics vol M18) (Berlin: Springer)

[8] Haake F 1973 Statistical Treatment of Open Systems by Generalized Master Equations (Springer Tracts of Modern Physics vol 66) (Berlin: Springer)

[9] Lax M 1963 Phys. Rev. 1292342

Lax M 1967 Phys. Rev. 157213

[10] Haake F and Reibold R 1985 Phys. Rev. A 322462

[11] Suarez A, Silbey R and Oppenheim I 1992 J. Chem. Phys. 975101 
[12] Gnutzmann S and Haake F 1996 Z. Phys. B 101263

[13] Gaspard P and Nagaoka M 1999 J. Chem. Phys. 1115668

[14] Barnett S M and Stenholm S 2001 Phys. Rev. A 64033808

[15] Wilkie J 2000 Phys. Rev. E 628808

Wilkie J 2001 J. Chem. Phys. 1147736

Wilkie J 2001 J. Chem. Phys. 11510335

[16] Budini A A 2004 Phys. Rev. A 69042107

[17] Daffer S, Wodkiewicz K, Cresser J D and McIver J K 2004 Phys. Rev. A 70 010304(R)

[18] Shabani A and Lidar D A 2005 Phys. Rev. A 71 020101(R)

[19] Chebotarev A M, Garcia J C and Quezada R B 1997 Math. Notes 61105

[20] Bardou F, Bouchaud J P, Aspect A and Cohen-Tannoudji C 2002 Lévy Statistics and Laser Cooling, (Cambridge: Cambridge University Press)

[21] Marte M A M and Zoller P 1989 Phys. Rev. A 405774

[22] Cresser J D and Pickles S M 1996 J. Opt. B: Quantum Semiclass. Opt. 873

[23] Herzog U 1995 Phys. Rev. A 52602

[24] Alemany P A 1997 J. Phys. A: Math. Gen. 306587

[25] Metzler R and Klafter J 2000 Phys. Rep. 3391

[26] Nirmal M, Dabbousi B O, Bawendi M G, Macklin J J, Trautmann J K, Harris T D and Brus L E 1996 Nature 383802

[27] Michler P, Imamoglu A, Mason M D, Carson P J, Strouse G F and Buratto S K 2000 Nature 406968

[28] Brokmann X, Hermier J P, Messin G, Desbiolles P, Bouchaud J P and Dahan M 2003 Phys. Rev. Lett. 90120601

[29] Aquino G, Palatella L and Grigolini P 2004 Phys. Rev. Lett. 93050601

[30] Kuno M, Fromm D P, Hamann H F, Gallagher A and Nesbitt D J 2001 J. Chem. Phys. 1151028

[31] Schlegel G, Bohnenberger J, Potapova I and Mews A 2002 Phys. Rev. Lett. 88137401

[32] Wagner M 1986 Unitary Transformations in Solid State Physics (Amsterdam: North-Holland) 